\title{
In vivo experimental intervertebral disc degeneration induced by bleomycin in the rhesus monkey
}

Fuxin Wei ${ }^{1 \dagger}$, Rui Zhong ${ }^{1 \dagger}$, Zhiyu Zhou ${ }^{1,2 \dagger}{ }^{1}$, Le Wang ${ }^{1}$, Ximin Pan $^{3}$, Shangbin Cui ${ }^{1}$, Xuenong Zou ${ }^{1}$, Manman Gao ${ }^{1}$, Haixing Sun ${ }^{3}$, Wenfang Chen ${ }^{4}$ and Shaoyu Liu ${ }^{1 *}$

\begin{abstract}
Background: Recently, biological therapies for early intervention of degenerative disc disease have been introduced and developed; however, a functional animal model that mimics slowly progressive disc degeneration of humans does not exist. The objective of this study was to establish a slowly progressive and reproducible intervertebral disc (IVD) degeneration model.
\end{abstract}

Methods: The subchondral bone adjacent to the lumbar IVDs (L3/4 and L5/6) of ten rhesus monkeys was randomly injected with $4 \mathrm{ml}$ bleomycin solution $(1.5 \mathrm{mg} / \mathrm{ml})$, or $4 \mathrm{ml}$ phosphate buffer saline (PBS) per segment as control, respectively. The degenerative process was investigated by using radiography and T1 $\rho$ MR imaging at 1, 3, 6, 9, 12 and 15 months postoperatively. Histological scoring, Sulfated Glycosaminoglycans (GAGs) analysis and real-time PCR were performed at 15 months. The correlation between histological score, GAGs and T1 $\rho$ values were also analyzed.

Results: The results showed that the mean T1 $\rho$ values of nucleus pulposus (NP) and annulus fibrosus (AF) in the bleomycin group significantly decreased after 3 and 6 months respectively, followed by slowly decrease until at 15 months. At 15 months, the histological scores was significantly higher, and the GAGs of NP was significantly lower in the bleomycin group, compared with the control group $(P<0.05)$. The results of real-time PCR revealed a significant increase in matrix metalloprotease (MMP)-3, A disintegrin and metalloproteinase with thrombospondin motifs (ADAMTS)-5, tumor necrosis factor $\alpha$, interleukin-1 $\beta$, interleukin- 6 expressions, transforming growth factor (TGF- $\beta 1$ ) and marked reduction in aggrecan, type II collagen, von willebrand factor (vWF) expressions at the mRNA levels in the bleomycin group. Spearman correlation analysis showed a strong positive correlation between GAGs and T1 $\rho$ values of NP $(r=0.740, P<0.01)$, and a significant inverse correlation between histological score and T1 $\rho$ values of NP and AF $(r=-0.761, r=-0.729$, respectively, $P<0.01)$.

Conclusions: Injection of bleomycin into the subchondral bone adjacent to the lumbar IVDs of rhesus monkeys can results in mild, slowly progressive disc degeneration, which mimics the onset of human disc degeneration. T1 p MR imaging is an effective and noninvasive technique for assessment of early stage disc degeneration.

Keywords: Intervertebral disc degeneration, Bleomycin, Animal model, Magnetic resonance imaging, Rhesus monkey

\footnotetext{
* Correspondence: gzsyliu@tom.com

'Equal contributors

'Department of Spine Surgery, the First Affiliated Hospital and Orthopedic

Research Institute of Sun Yat-sen University, Guangzhou, China

Full list of author information is available at the end of the article
} 


\section{Background}

Intervertebral disc (IVD) degeneration,which is an epidemic human condition with a major socioeconomic impact, is believed to be a main cause of low back pain [1]. However, a clear understanding of the basic mechanisms of disease pathogenesis and specific therapeutic agents is still limited [2]. An experimental animal model of human IVD degeneration is needed. It is well known that IVD is the largest avascular structure in the human body that has limit the capacity for regeneration. Although there are several animal models of experimental disc degeneration that have been developed, which including spontaneous degeneration models [3], annulus fibrosus injury models [4], and chemically induced models et al. [5], these methods could not model the slowly ischemic and progressive disc degeneration of human beings effectively. Although the needle puncture methods have been recently developed and used to induce disc degeneration models to test the effectiveness of new therapies for reversing IVD degeneration [4], this method induces to IVD degeneration through direct injury, which is not easy to control the degenerative process. Furthermore, these models are not suitable for the studies related to biological treatment of IVD degeneration to some extent.

The nutritional pathways that into the nucleus pulposus of human intervertebral discs are mainly by diffusion through the central portion of the end-plate from these marrow space cartilage contacts and diffusion through the annulus fibrosus from the surrounding vessels [6]. An alternation of the nutrient pathway is considered as one of the main causes of IVD degeneration [7]. Bleomycin, which have been used as sclerosing agents, has proved to be safe and effective in the treatment of vascular malformations of the head and neck $[8,9]$. Intralesionally injected bleomycin brings the drug into direct contact with the endothelial lining and destroys the endothelial cells, resulting in sclerostenosis of the lumen and leading to narrowing or occlusion of the vessels [10].

The purpose of this study was to establish an early ischemic, progressive, and reproducible IVD degeneration model induced by injection of bleomycin into subchondral bone adjacent to the intervertebral disc of rhesus monkeys to provide a basis for further in vivo studies on IVD degeneration. Combined with histology, real-time PCR and Sulfated Glycosaminoglycans (GAGs) assessment, the T1 $\rho$ magnetic resonance imaging (MRI) technique [11,12], which has been reported strongly correlated with proteoglycan content were performed to evaluate the progression of IVD degeneration.

\section{Methods}

\section{Animals}

Ten rhesus monkeys (six females and four males), with a mean age of $5.32 \pm 0.47$ years old (range, $5-7$ years), which can be converted to equivalent human age by using a 1:3.5 ratio [13], and a mean body weight of $6.83 \pm 0.67 \mathrm{~kg}$ (range, $5.2-7.5 \mathrm{~kg}$ ) were used in this study. All of the animals were provided by the center of Guangdong Landao Biotechnology in Guangzhou of China, and exclude spinal deformity by radiological examination. The study protocol was reviewed and approved by the institutional review board and ethics committee of the First Affiliated Hospital of our university (No. 2010-204). The animal study was also approved by the institutional review board and animal care committee of the First Affiliated Hospital of our university (2013A-204).

\section{Animal groups}

In this study, a single concentration of $1.5 \mathrm{mg} / \mathrm{ml} \mathrm{bleo-}$ mycin in phosphate buffered saline (PBS) was performed. Unlike human beings, there are 7 intervertebral discs (IVDs) in rhesus monkeys. The subchondral bone adjacent to the 2 lumbar IVDs (L3/4 and L5/6) were randomly injected with $4 \mathrm{ml}$ bleomycin solution (Bleomycin, $\mathrm{n}=10$ ), or $4 \mathrm{ml}$ PBS (Control, $\mathrm{n}=10$ ), respectively. The degenerative process was investigated by using radiography, T1 $\rho$ MR imaging for 15 months.

\section{Experimental surgery}

All the experimental surgeries were performed in the orthopedic research institute of Sun Yat-sen University. Intramuscular injections of $10 \mathrm{mg} / \mathrm{kg}$ ketamine and $0.5 \mathrm{mg} /$ $\mathrm{kg}$ midazolam were used for anesthesia of the monkeys, followed by $0.3 \mathrm{mg} / \mathrm{kg}$ midazolam and $4 \mu \mathrm{g}$ fentanyl per hour. Under sterile surgical conditions, the vertebral bodies from L3 to L6 were exposed by using a left retroperitoneal approach. After clearly identified, the subchondral bone adjacent to the IVDs of L3-4 and L5-6 was performed according to the preoperative design. To induce the subendplate ischemic model, a hole $1.5 \mathrm{~mm}$ in diameter and $15 \mathrm{~mm}$ in depth was drilled in the middle of the intervertebral body approximately $1.5 \mathrm{~mm}$ above and below the disk, followed by slow injection of $2.0 \mathrm{ml}$ bleomycin $(1.5 \mathrm{mg} / \mathrm{ml}$, Tianjin Taihe Pharmaceutical, Tianjin, People's Republic of China) per hole for the belomycin group, and the same volume of PBS for the control group, respectively. Then, each hole was sealed with bone wax.

Before, during, and after operation, the monkeys were given $80 \mathrm{mg}$ per kilogram of ceftriaxone sodium (Baiyun Pharmaceuticals, Guangzhou, China) subcutaneously. After operation, the monkeys were housed individually with free access to water. The rhesus monkeys tolerated general anesthesia well and no mortalities from complications caused by anesthesia or infections were found among the animals. Weight, food intake, and sleeping habits were recorded. 


\section{IVD height measurement}

The lateral views of roentgenograms for the lumbar spine were acquired for all the monkeys by using the Digital Diagnost VM (Philips, Best, the Netherlands), a multipurpose single-detector digital radiography system. The average IVD height was measured manually by an orthopedic researcher (R. Z.) who was blinded to both the injection solution used (PBS/Belomycin) and the follow-up period. The average IVD height was calculated by averaging 3 measurements (Figure 1A). The average IVD height was obtained concordantly. Subsequently, the average IVD height was divided by the average caudal vertebral height, resulting in a value called the disc height index (DHI) leveling out interanimal size difference [14]. Changes in the DHI were expressed as \%DHI and normalized to the measured preoperative DHI (\%DHI = postoperative $\mathrm{DHI} /$ preoperative $\mathrm{DHI} \times 100 \%)$ [15].

\section{MR imaging analysis}

Each animal underwent MRI on a 1.5-T MR imager (Achieva, Philips Healthcare, Best, The Netherlands) with a supine position on a spine-array coil $(5 \times 11$ inches). The T1 $\rho$-weighted images were acquired by using spin-lock pulses followed by a spin-echo acquisition, with time of spin-lock of 2, 15, 30, and $45 \mathrm{msec}$ and spin-lock frequency of $250 \mathrm{~Hz}$. Using the Siswin software, we mapped these data and exported the data to mapping images. Article by Zhou et al. contains details of the T1p sequence [16]. The T1 $\rho$ values were calculated on the basis of selection of the regions of interest (ROIs) [16], which were manually drawn over the $\mathrm{T} 1 \rho$ maps of the discs by a radiologists (X.P.) with more than 5 years' experience reading

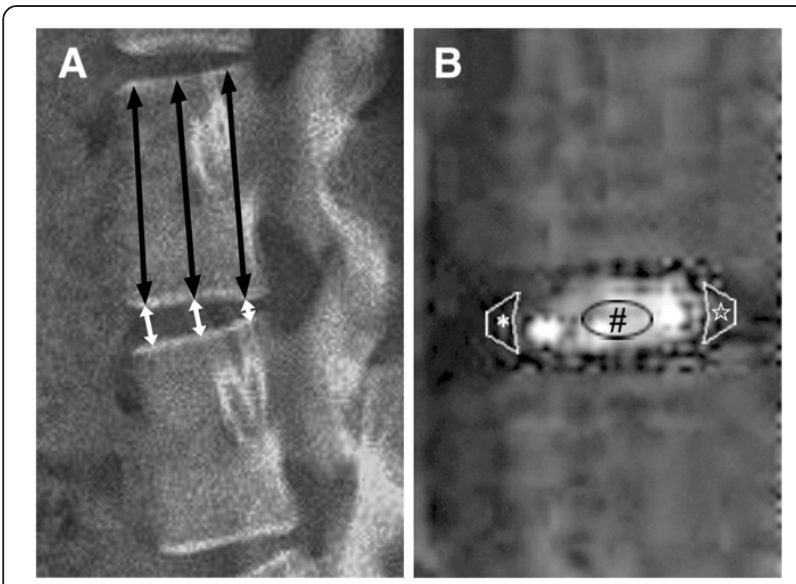

Figure 1 Radiography and T1 $\rho$-weighted images of a part of the lumbar spine of rhesus monkeys. (A) The Disc Height Index was calculated by dividing the average disc height (white arrows) by the average vertebral body height (blasck arrows). (B) An example of placement of ROls over $\mathrm{NP}(\#)$, anterior $A F(*)$ and posterior $\mathrm{AF}(\hat{\boldsymbol{\gamma}})$ was showed in the IVD respectively. ROls: regions of interest; NP: nucleus pulposus; AF: annulus fibrosus; IVD: Intervertebral disc.
MRI images. ROIs included nucleus pulposus (NP), anterior annulus fibrosus (AF) and posterior AF (Figure 1B). Values of anterior and posterior AF were averaged as the value for AF. To evaluate ROIs in a standardized and reproducible way, we decided to measure oval ROIs $\left(0.1 \mathrm{~cm}^{2}\right.$ on NP, $0.05 \mathrm{~cm}^{2}$ on AF). The average of three mid-sagittal values from one disk was used for the statistical analysis.

\section{Tissue harvesting}

Disc tissues, which involved adjacent endplates, were harvested from all the rhesus monkeys under anesthesia with intramuscular injections of $10 \mathrm{mg} / \mathrm{kg}$ ketamine and $0.5 \mathrm{mg} / \mathrm{kg}$ midazolam, at 15 months after surgery. All the specimens were dissected into two halves in the sagittal plane, one half was fixed for histology analysis, and the other half was sub-dissected into another two halves, which were used for messenger RNA (mRNA) analysis and Sulfated Glycosaminoglycans (GAGs) assessment.

\section{Histology evaluation and histological scores}

The specimens were fixed with $4 \%$ paraformaldehyde for 24 hours at $4^{\circ} \mathrm{C}$, then transferred to a sealed vial containing a solution of $70 \%$ ethanol and decalcifying agent for 30 days. After washed with water, the specimens were sequentially dehydrated, split down the mid-sagittal plane, embedded in paraffin for histology sectioning. Serial sections were cut in the transverse plane at $8 \mu \mathrm{m}$ with a microtome (Microtome International, Waldorf, Germany), and then stained with hematoxylin and eosin (H\&E), masson trichrome or safranin O staining for light microscopic examination.

The histological changes were qualitatively analyzed by a pathologist (W.CH.) using a digital image analysis system (Nikon Eclipse Ti; Nikon, Tokyo, Japan) who was blinded to the different treatments between groups, according to the classification scale described by Masuda et al. [4]. The criteria are summarized in Table 1 . The histological score is the sum of the scores of the 3 individual parameters and ranged from 3 to 9 , where normal is 1point for each of the 3 categories listed above, for a total of 3 points. A total of 9 points is representative of severe degeneration.

\section{Sulfated Glycosaminoglycans (GAGs) assay}

NP from the other half samples were digested using papain at $65^{\circ} \mathrm{C}$ for 2 hours. GAG content was calculated in duplicate by dimethylmethylene blue (DMMB) procedure [17], using chondroitin-4-sulfate (Sigma-27042) as a standard. The DNA concentration of each sample was measured using the PicoGreen assay (Molecular Probe) and used for normalizing the GAG values.

\section{Real-time PCR analysis}

Total RNA were extracted from the specimens using Trizol reagent (Ambion, Carllsbad, CA, USA), followed by the 


\section{Table 1 Degeneration histologic grading scale}

\section{Anulus fibrosus}

Grade:

$1 \quad$ Normal, pattern of fibrocartilage lamellae without ruptured fibers and without a serpentine appearance anywhere within the anulus

2 Ruptured or serpentine patterned fibers in less than 30\% of the anulus

3 Ruptured or serpentine patterned fibers in more than $30 \%$ of the anulus

II Border between the anulus fibrosus and nucleus pulposus

Grade:

$1 \quad$ Normal

$2 \quad$ Minimally interrupted

3 Moderate/severe interruption

III Cellularity of the nucleus pulposus

Grade:

1 Normal cellularity with large vacuoles in the gelatinous structure of the matrix

2 Slight decrease in the number of cells and fewer vacuoles

3 Moderate/severe decrease (>50\%) in the number of cells and no vacuoles

Table 2 Sequences of primers used in the real-time PCR

\begin{tabular}{|c|c|c|c|}
\hline Name & GeneBank accession No. & & Sequence $\left(5^{\prime}-3^{\prime}\right)$ \\
\hline \multirow[t]{2}{*}{ GAPDH } & XM_001091567.2 & Forward & ACAATCCCATCACCATCTCGC \\
\hline & & Reverse & GACCCTITTGGCCTCCTCATC \\
\hline \multirow[t]{2}{*}{ Aggrecan } & XM_002804944.1 & Forward & ACTCGCTGAGTGTCAGCATC \\
\hline & & Reverse & ACACACGGCTCCACTTGATT \\
\hline \multirow[t]{2}{*}{ Collar } & XM_001096194.2 & Forward & CCAGCCGCAAAGAGTCTACA \\
\hline & & Reverse & TGGTGGGATGTCTTCGTCTTG \\
\hline \multirow[t]{2}{*}{ Col2a1 } & XM_001100559.2 & Forward & GTGTCAGGGCCAGGATGTC \\
\hline & & Reverse & AGGGGCACCTIITTCACCTT \\
\hline \multirow[t]{2}{*}{ MMP-3 } & XM_001098400.2 & Forward & GGCGCAAATCTCTCAGGAAG \\
\hline & & Reverse & GGCCCAGAACTGATTTCCTIT \\
\hline \multirow[t]{2}{*}{ ADAMTS-5 } & XM_001103439.2 & Forward & GTGGCTCACGAAATCGGACA \\
\hline & & Reverse & GGTGGCTGAAGTGCATTTGG \\
\hline \multirow[t]{2}{*}{ vWF } & NM_001243086.1 & Forward & GAGGGTGGTTGGTGGATGTC \\
\hline & & Reverse & CTAGGCCATGCTCCTAGCTG \\
\hline \multirow[t]{2}{*}{ TNF-a } & NM_001047149 & Forward & CCCCAAGGACCCCTCTCTAA \\
\hline & & Reverse & GGGTTTGCTACAACATGGGC \\
\hline \multirow[t]{2}{*}{$\mid L-1 \beta$} & NM_001042756 & Forward & GACGTCGATGGCCCTAAACA \\
\hline & & Reverse & AAGCCCTCGTTGTAGTGCTC \\
\hline \multirow[t]{2}{*}{ IL-6 } & NM_001042733 & Forward & GGTACATCCTCGACGGCATC \\
\hline & & Reverse & CCAGGCAAGTGTCCTCATTG \\
\hline \multirow[t]{2}{*}{ Caveolin 1} & NM_001168614 & Forward & ACGTAGACTCGGAGGGACAT \\
\hline & & Reverse & AGCGATGGTGATTCCCCAAG \\
\hline \multirow[t]{2}{*}{ TGF $\beta 1$} & XM_001100842 & Forward & GGGCTACCATGCCAACTTCT \\
\hline & & Reverse & CCAGGACCTTGCTGTACTGT \\
\hline
\end{tabular}

RNeasy Mini Kit (Qiagen Inc., Duesseldorf, Germany). Reverse transcription was performed at $42^{\circ} \mathrm{C}$ for 50 minutes using the SuperScript First-strand Synthesis Kit (TOYOBO, Biotech, Co., Ltd., Shanghai, China). Aggrecan, type Icollagen (Col1 $\alpha 1)$, type IIcollagen (Col2 $\alpha 1)$, Matrix metalloproteinases (MMP)-3, A disintegrin and metalloproteinase with thrombospondin motifs (ADAMTS)-5, tumor necrosis factor $\alpha$ (TNF $\alpha)$, interleukin-1 $\beta$ (IL-1 $\beta)$, interleukin-6 expressions (IL-6), caveolin- 1 , transforming growth factor- $\beta 1$ (TGF- $\beta 1$ ) and glyceraldehyde 3-phosphate dehydrogenase (GAPDH) gene expression of the intervertebral discs were quantified by real-time PCR using CFX96 Real-Time System (Bio-Rad, Herculus, CA, USA). The sequence of all the primers used for real-time PCR are shown in Table 2. With a serially-diluted cDNA sample mixture, a positive standard curve for each primer was obtained for real-time PCR. Quantitations of gene expression of aggrecan, Col $1 \alpha 1$, Col2 $\alpha 1$, MMP-3, ADAMTS-5, TNF $\alpha$, IL-1 $\beta$, IL-6, caveolin1 and TGF- $\beta 1$ in the intervertebral discs were calculated using standard curves and normalized to GAPDH in each specimen. In order to evaluate the number of capillaries in the cartilage endplate, the quantitation of gene expression of von willebrand factor (vWF) in the bone endplates was 
also calculated using the same method as mentioned above. The experiments were repeated at least twice for enhanced accuracy.

\section{Statistical analysis}

SPSS 16.0 software (SPSS Inc., Chicago, IL) was used for univariate analysis of variance. The data within groups were analyzed using One-way Analysis of Variance (ANOVA) and Fisher's protected least significant difference test. The data between groups were compared by using paired $t$ test. The correlation between the \%DHI, histological score, GAGs content, and T1 $\rho$ values of NP and AF were assessed by the Spearmann rank correlation test. Data are presented as the mean \pm standard deviation. Statistical significance was indicated at $P<0.05$.

\section{Results}

No postoperative morbidity or mortality was recorded. All animals recovered uneventfully after surgery and quickly resumed normal activities in the cage. None of these animals showed any remarkable change in weight, eating patterns, or sleeping habits.

\section{Intervertebral disc height}

In the control groups, the DHI changes were not significant at any time point examined. In contrast, the DHI in the bleomycin group progressively decreased over time (Figure 2A), reaching significance when compared with that in the control group after 12 months $(P=0.02)$, and was followed by slowly declining. After 15 months, the DHI decreased by $12.9 \%$, which was not significant, compared with the DHI at the 12 -month time point $(P=0.76)$.

\section{Histological findings and GAGs assay}

The intervertebral discs in the control group appeared normal. The annulus fibrosus showed normal organization of fibrocartilage lamellae (Figure 3A), and the nucleus pulposus contained abundant cells and sounded by large zones of acellular matrix (Figure 3B,C). In the bleomycin group, the discs showed degenerative changes, where the nucleus pulposus comprised relatively few cells (Figure 3E) and less proteoglycans (Figure 3F), relative to the control group. The annulus fibrosus showed less organized fibrocartilage lamellae, and the collagen fibers formed a wavy arrangement (Figure 3D) in the bleomycin group.

The histological score of the discs in the bleomycin group was significantly higher than the control groups $(P<0.01$, Figure $4 \mathrm{~A})$. The total GAG of NP in the bleomycin group decreased significantly compared with the control group $(P<0.01$, Figure 4B).

\section{MRI}

The MRI images showed degenerative signs in IVDs injected with bleomycin (Figure 5). There were no significant differences in $\mathrm{T} 1 \rho$ values of NP and AF in the control group at any time point. In the belomycin group, the T1 $\rho$ values of NP and AF slowly declined after operation. After 3 months, the decrease in NP was significant $(P=0.02$, Figure $2 \mathrm{~B})$. However, the decrease in AF did not show any significant difference, until 6 months after operation (Figure $2 \mathrm{C}$ ). Interestingly, the $\mathrm{T} 1 \rho$ values of NP showed a rapid decrease during the second 3 months from $109.3 \mathrm{msec} \pm 10.8$ to $82.6 \mathrm{msec} \pm 11.9(P=0.003)$, in the bleomycin group, and then decreased slowly. After 12 months, both decrease of $\mathrm{T} 1 \rho$ values in NP and AF tended to be stable.

The correlation of T1 $\rho$ values to \%DHI, histological score, or GAGs content was assessed by the Spearman rank correlation test. Although both of the T1 $\rho$ values of $\mathrm{NP}$ and AF negatively correlated with the histological score, and positively correlated with the total NP GAGs significantly $(P<0.05$, Figure 6 , Table 3$)$, the T1 $\rho$ values of AF did so more weakly than that of NP. However, the
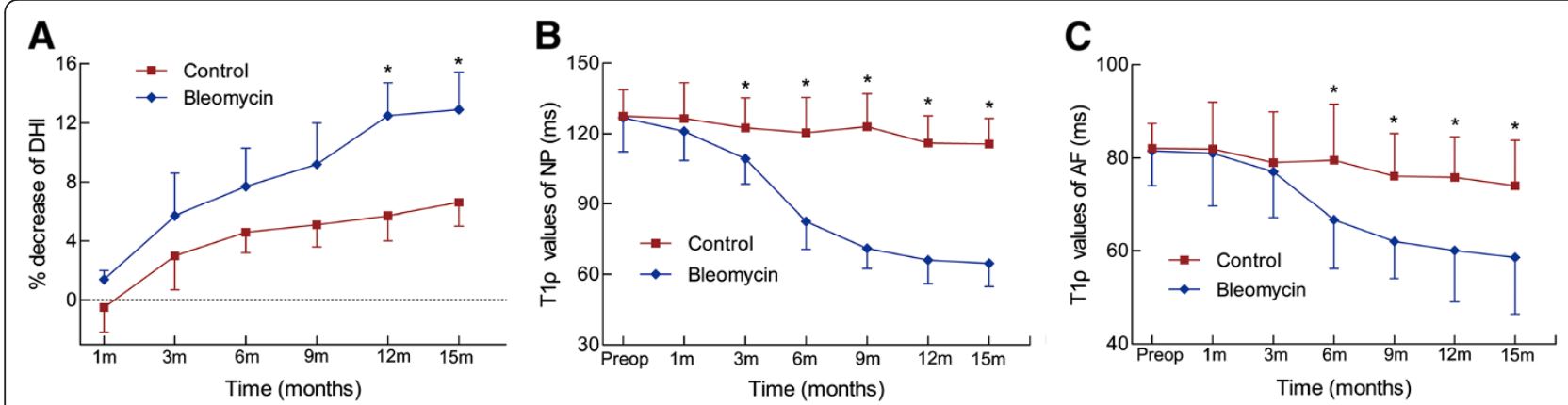

Figure 2 Mean \%DHI, T1 $\rho$ values of NP and AF at each time point. (A) A significant decrease of \%DHI was observed at 12 months postoperatively in the bleomycin group $\left({ }^{*} P<0.05\right)$. (B) A significant decrease in the T1 $\rho$ values of NP was observed at 3 months postoperatively in the bleomycin group ( $\left.{ }^{*} P<0.05\right)$, followed by a rapid decrease during the second 3 months. (C) A significant decrease in the T1 $p$ values of AF was observed at 6 months postoperatively in the bleomycin group $\left({ }^{*} P<0.05\right)$, followed by slowly decrease. DHI: Disc height index; NP: nucleus pulposus; AF: annulus fibrosus. 


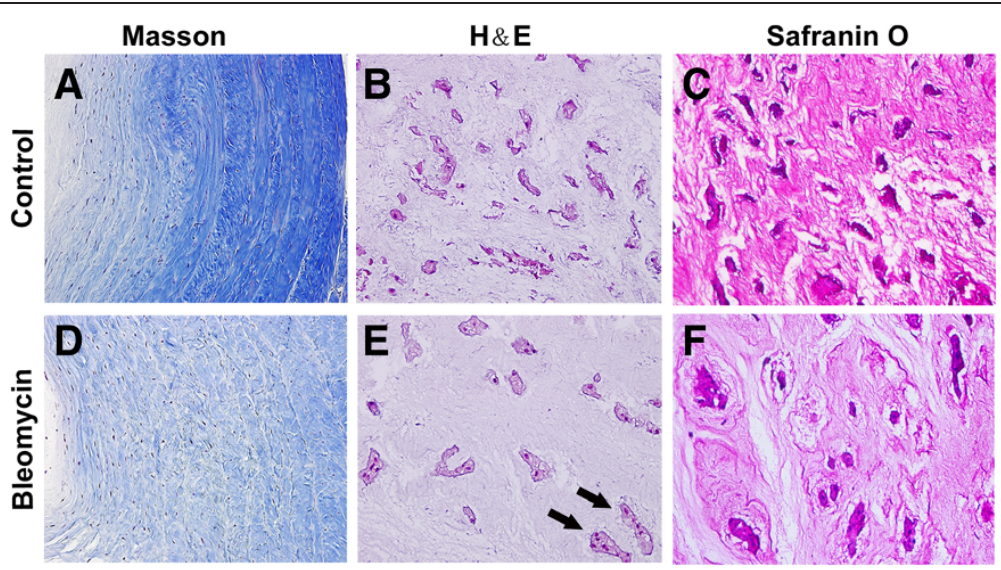

Figure 3 Hematoxylin and eosin (H\&E), masson trichrome and safranin $\mathrm{O}$ staining for NP and AF. (A)-(C) The annulus fibrosus showed normal organization of fibrocartilage lamellae, and the nucleus pulposus contained abundant cells and sounded by large zones of acellular matrix in the control group. (D)-(F) In the bleomycin group, the nucleus pulposus comprised relatively few cells and less proteoglycans, combined with less organized fibrocartilage lamellae and a wavy arrangement of collagen fibers, relative to the control group. Black arrows indicate notochordal cells based on morphology.

positive correlation between the $\mathrm{T} 1 \rho$ values of $\mathrm{AF}$ and the \%DHI was much stronger than that of NP (Table 3).

\section{Real-time PCR}

The expression of Aggrecan and Col2 $\alpha 1$ were significantly lower, and the expression of MMP-3, ADAMTS-5, TNF- $\alpha$, IL-1 $\beta$, IL- 6 and TGF- $\beta 1$ were significantly upregulated in the bleomycin group as compared with the control group $(P<0.05$, Figure 7$)$. The expression of vWF in the bleomycin group significantly decreased in comparison with the control groups $(P<0.05$, Figure 7$)$. However, there were no significant differences in the expression of Col1 $\alpha 1$ and caveolin-1 between groups ( $P>0.05$, Figure 7$)$.

\section{Discussion}

It has been generally recognized that degenerative disc disease is the most common cause of low back pain. Recently, biological therapies for early intervention of degenerative disc disease have been introduced and developed, including direct delivery of specific growth factors, plate-rich plasma, adenovirus particles or autologous cells, into the disc [18-20]. Thus, it is essential to establish a suitable disc degenerative model that could better model the human disease process to evaluate the novel treatment options. However, to our known, there are no particular models currently parallel the complex nature of human disc degeneration $[17,21]$. In the present study, we designed a new method of inducing an IVD degeneration model in the rhesus monkey and have demonstrated that it could induce slowly progressive and mild disc degeneration, which mimicked the onset of human disc degeneration.

It is known that the nutrient exchange for disc cells is mostly from the capillaries of the subchondral plate of the vertebral body, across the layers of hyaline cartilage that constitute the endplate [22]. Disorders that affect the blood supply to the endplate are significantly associated with disc degeneration and back pain [23]. In our study, we injected bleomycin,which could produce a "devascular effect" [8,9], into the subchondral bone adjacent to the IVDs of rhesus monkeys. The results of quantitative T1 $\rho$ MR imaging showed that the T1 $\rho$ values of nucleus pulposus and annulus fibrosus decreased slowly after surgery, compared with the control group. The degenerative signs of this model developed slower, compared with other animal models, such as needle puncture models or chemically induced models, which were reported to degenerate significantly within 2 to 4 weeks [21]. However, as disc degeneration develops in several decades in humans, the slow development of the degeneration in the rhesus model is relative. In this study, another interesting finding was that, the results showed a great decrease in $T 1 \rho$ values of $\mathrm{NP}$ in the second 3 months after surgery. This was similar to the degenerative process of human subjects, which had also showed a rapid decrease in T1 $\rho$ values between Pfirrman gradesIIand III reported by Zhou et al. [16]. This, to some extent, suggested that the degenerative process of this model may be a better surrogate used for investigation of early stage disc degeneration and regeneration studies.

The histomorphological findings and GAGs assay showed that the GAGs in nucleus pulposus decreased significantly in the bleomycin group. These changes were consistent with the previous studies showing loss of proteoglycans during the initial phase of disc degeneration [24]. In human discs, the notochordal cells (NCs) are lost during adolescence, which is also when discs begin to show degenerative signs [25]. Although the cell population significantly decreased in nucleus pulposus of this degenerative model of rhesus monkeys, notochordal cells were still observed. This 


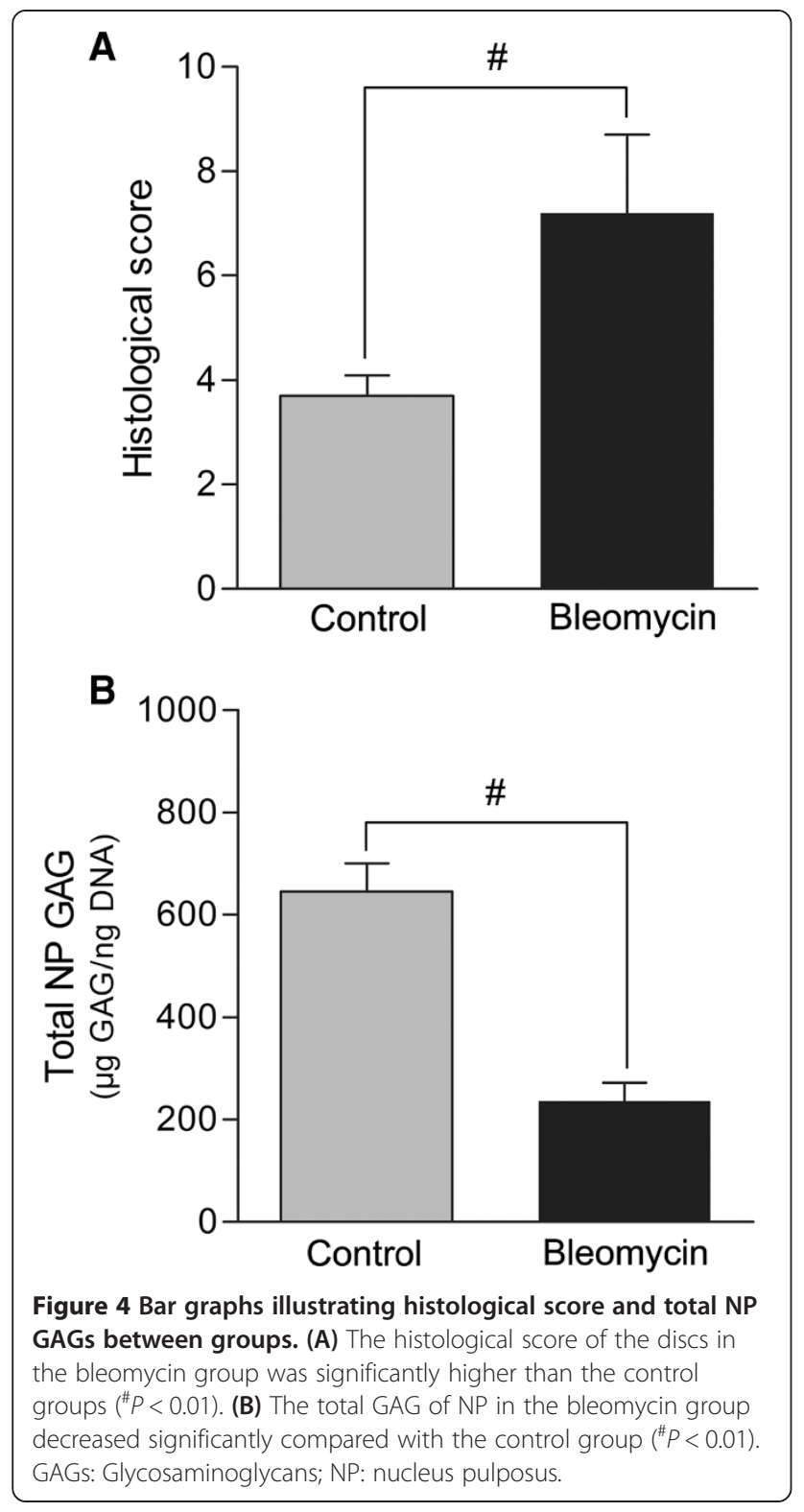

may be related to the age of the rhesus monkeys. Although this limits relevance to humans, these changes in the degenerative model of this study confirmed that injection of bleomycin into the subchondral bone adjacent IVD of rhesus monkeys could induce early disc degeneration, which showed some similar characteristic features to the complex processes of disc degeneration in human, such as disc height loss, significant decrease of cell population and glycosaminoglycans in the nucleus pulposus, and less organized fibrocartilage lamellae in the annulus fibrosus, et al.

As previously reported, the histological scale and radiographic findings for assessing IVD degeneration showed a consistency to MRI evaluation [4]. In this study, with Sperman rank correlation coefficient of -0.761 and -0.729 $(P<0.01)$, the correlation between the histological scores and the T1 $\rho$ values of NP and AF are significant. Although the correlation between the \%DHI and the T1p values of NP and AF are also significant, but not so strong. This may be due to the different sensitivity of histological and disc height analyses in response to disc degeneration. Early disc degeneration with a slight loss of nucleus pulposus that may be easily detected by histology, may not show significant differences in the disc height change, which is largely affected by the structural changes. It has been reported that T1 $\rho$ MRI imaging is strongly correlated with proteoglycan content $[11,12]$. This is also observed in our study, which showed strong correlation between the total NP GAGs and the T1 $\rho$ values of NP and AF.

It has been revealed that proteoglycans has the ability to bind to collagen, growth factors, and other matrix components [26]. In this study, it has been shown that the collagenIIexpression in the discs of bleomycin group was reduced compared with that in the control group, which was consistent with the theory that proteoglycans can bind to collagens. The degenerative changes in the IVDs, such as composition of extracellular matrix (ECM), loss of disc cells, proteoglycan and water content, have been suggested to be the consequence of an up-regulation of catabolic MMPs and two major aggrecanases, ADAMTS- 4 and 5 $[27,28]$. Consistent with the previous reports $[27,28]$, we found that mRNA expression of MMP-3 and ADAMTS-5 were significantly increased in the discs of bleomycin

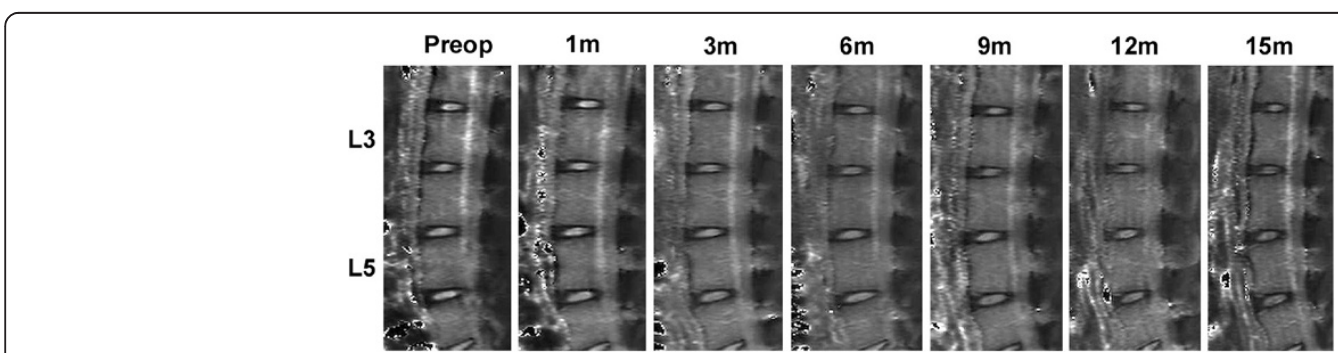

Figure 5 The representative T1 $\rho$ maps of lumbar spine in an rhesus monkey model. Bleomycin and PBS solution was injected at L3-4 and L5-6 respectively. Preop: Preoperatively. 


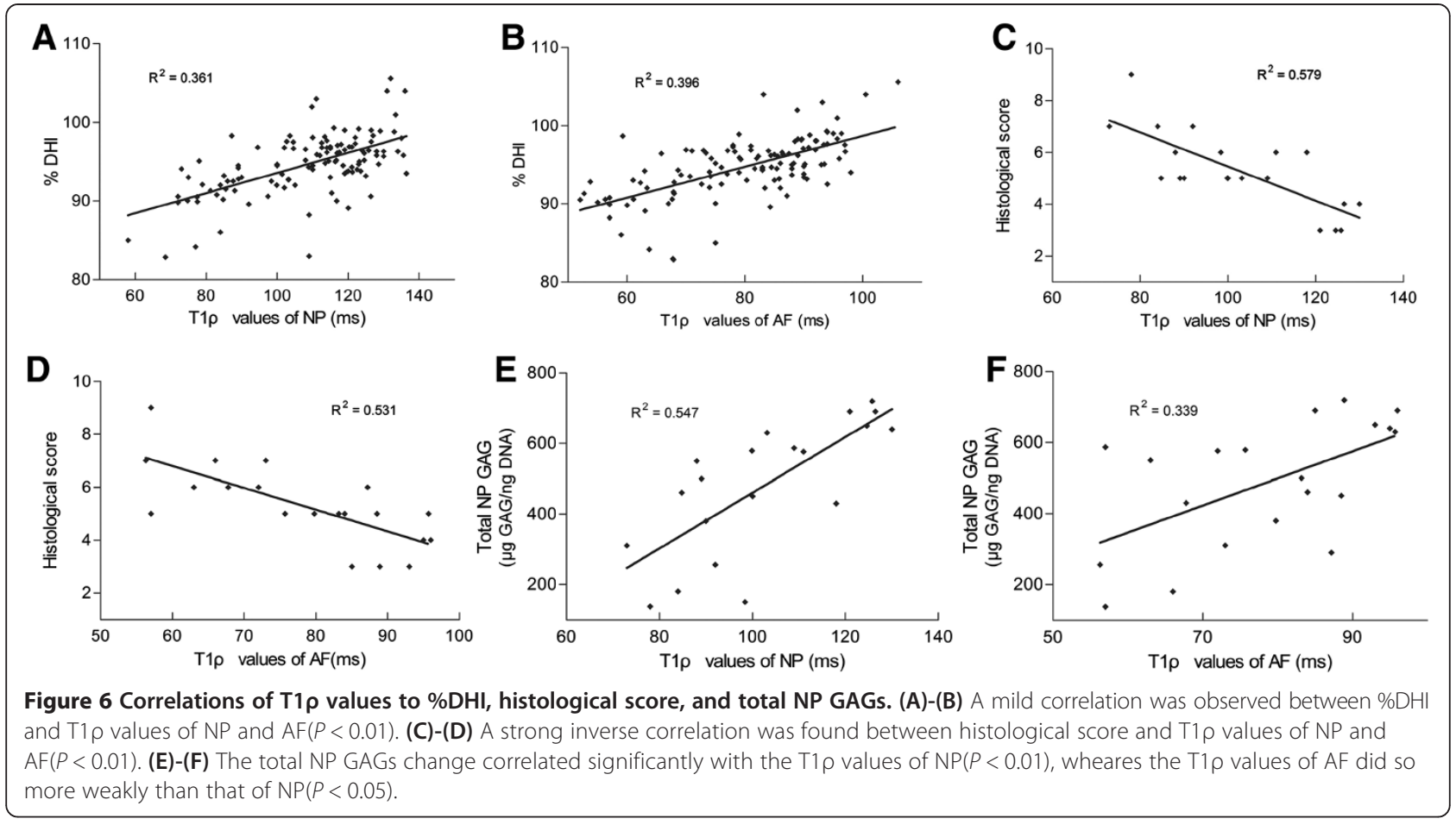

group compared to the control group. This may be due to the abnormal nutrient transport between the IVD and endplates due to the "devascular effect" of bleomycin, which was confirmed by the mRNA expression of vWF in the bone endplates.

It has been reported that the cytokines TNF- $\alpha$, IL- $1 \beta$, IL-6 and IL-8 increased significantly in aged and/or degenerated IVDs [29-31]. In this study, there were significant increase of the gene expression of TNF- $\alpha$, IL-1 $\beta$ and IL- 6 in the IVD of bleomycin group. It has been confirmed that hypoxia could increases oxidative stress in several cell types [32,33], including the notochordal intervertebral disc cells [34]. In this study, the nutrition exchange of the IVD was disturbed by injection of bleomycin into the subchondral bone adjacent to the IVDs of rhesus monkeys. This could disturb the oxygen supply to the IVDs, and then induced increasing oxidative stress, which might account for the

Table 3 The correlation among disc height index, histological score, total NP GAGs and T1 $\rho$ values (Sperman rank correlation coefficient, rho)

\begin{tabular}{llll}
\hline & $\%$ DHI & Histological score & Total NP GAGs \\
\hline T1 $\rho$ values of NP & 0.601 & -0.761 & 0.740 \\
& $P<0.0001$ & $P<0.0001$ & $P<0.0001$ \\
T1 $\rho$ values of AF & 0.630 & -0.729 & 0.582 \\
& $P<0.0001$ & $P=0.0003$ & $P=0.02$ \\
\hline
\end{tabular}

$\mathrm{NP}=$ nucleus pulposus; $\mathrm{AF}=$ annulus fibrosus; $\% \mathrm{DHI}=$ change in disc height index.

GAGs = glycosaminoglycans. increasing gene expression of inflammatory cytokines in the IVDs.

TGF- $\beta$ is a multifunctional regulator of cellular proliferation, differentiation, and extracellular matrix (ECM) production. Thompson et al. [35] reported the anabolic effect of TGF- $\beta$ on proteoglycans (PGs) synthesis in canine disc cells. Lee et al. [36] found that the level of TGF- $\beta$ was higher in patients with disc degenerative

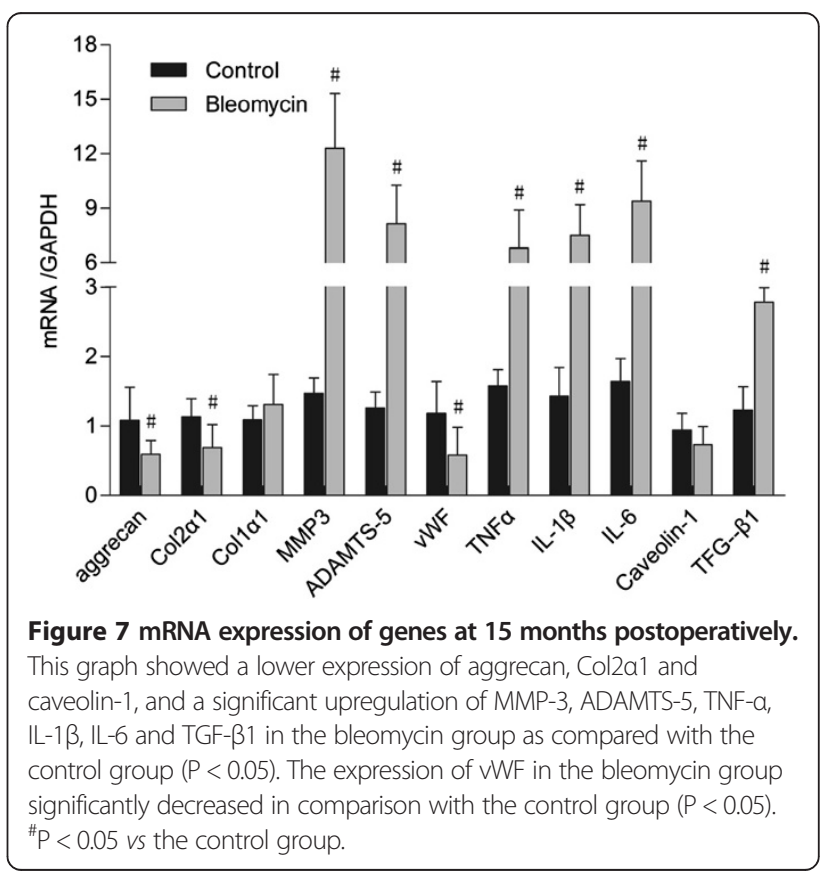


disease (DDD). However, it has also been reported that overexpression of TGF- $\beta$ showed deleterious effect on degenerated cartilage tissue via increase of aggrecanase1 and MMP-13 $[37,38]$. In this study, the gene expression of TGF- $\beta$ was more higher in the degenerative discs, which was consistent with the previous reports $[35,36]$. Caveolin-1, which is a scaffold protein of caveolae, is elevated in degenerative discs and has been proposed to play a prominent role in the pathogenesis of IVD degeneration [39]. However, Smolder et al. [40] found that IVD degeneration involved significant downregulation in caveolin-1. In this study, the gene expression of caveolin-1 was down-regulated in the degenerative discs. This may be due to the effect of bleomycin, which can lead to caveolin-1 down-regulation in fibrosing lung [41]. No matter what reason it is, however, further studies are warranted to evaluate the role of caveolin-1 in disc degenerative disease.

In our study, rhesus monkeys, higher in the phylogenetic tree, were used because of the similarities of their anatomic and physiological characteristics, and IVD anatomy similar to that of humans [42]. Thus, this ischemic degenerative model in the present study could better simulate the IVD degeneration of humans. However, this study also has some limitations. We did not perform the histological evaluation during the follow up period, due to the high cost and limited number of animals. Another limitation was that we did not perform the biomechanical evaluation, such as hydrostatic pressure in the degenerative discs. Additional studies are warranted to further evaluate the mechanism and characters of disc degeneration induced by bleomycin.

\section{Conclusions}

This current study demonstrate that the injection of bleomycin into the subchondral bone adjacent to the lumbar IVDs of rhesus monkeys can induce slowly progressive and mild disc degeneration, which mimics the onset of human disc degeneration. T1 $\rho$ MR imaging is an effective and noninvasive technique for assessment of disc degeneration. The degeneration model is suitable for disc degeneration and regeneration studies. Further studies to fully establish this model, however, are needed.

\footnotetext{
Abbreviations

IVD: Intervertebral disc; GAGs: Glycosaminoglycans; DHI: Disc height index; ROls: Regions of interest; NP: Nucleus pulposus; AF: Annulus fibrosus; H\&E: Hematoxylin and eosin; DMMB: Dimethylmethylene blue; MMP: Matrix metalloproteinases; ADAMTS: A disintegrin and metalloproteinase with thrombospondin motifs; GAPDH: Glyceraldehyde 3-phosphate dehydrogenase; Col1: Type Icollagen; Col2: Typellcollagen; vWF: Von willebrand factor; TGF: Transforming growth factor; DDD: Disc degenerative disease.
}

\section{Competing interests}

The authors declare that they have no competing interests.

\section{Authors' contributions}

FW, ZZ, SL, LW and SC performed experimental surgery. RZ, HS and XP performed radiological and MRI evaluation. W.CH. performed histological evaluation. RZ performed PCR analysis. SL and XZ conceived of the study and participated in its design. FW drafted the manuscript. MG performed the statistical analysis. All authors read and approved the final manuscript.

\section{Acknowledgements}

This study was founded by National Natural Science Foundation of China (No. 81401839, U1032001), Science \& Technology support Project of Huangpu (201329-04), Science \& Technology Planning Project of Guangdong Province (No. 2010B010800019) and Natural Science Foundation of Guangdong (No. S2013010015775).

We thank Steffen Ringgaard for technical assistance of T1 $\rho$ imaging, and Anthony N. Khoury who helped to copyedit the paper to improve the style of written English.

\section{Author details}

${ }^{1}$ Department of Spine Surgery, the First Affiliated Hospital and Orthopedic Research Institute of Sun Yat-sen University, Guangzhou, China. ${ }^{2}$ The medical school of Shenzhen University, Shenzhen, China. 'Department of Radiology, the First Affiliated Hospital of Sun Yat-sen University, Guangzhou, China. ${ }^{4}$ Department of Pathology, the First Affiliated Hospital of Sun Yat-sen University, Guangzhou, China.

Received: 15 June 2014 Accepted: 3 October 2014

Published: 9 October 2014

\section{References}

1. Wang C, Gonzales S, Levene H, Gu W, Huang CY: Energy metabolism of intervertebral disc under mechanical loading. J Orthop Res 2013, 3:1733-1738.

2. Hughes SP, Fremont AJ, Hukins DW, McGregor AH, Roberts S: The pathogenesis of degeneration of the intervertebral disc and emerging therapies in the management of back pain. J Bone Joint Surg Br 2012, 94:1298-1304.

3. Bergknut N, Rutges JP, Kranenburg HJ, Smolders LA, Hagman R, Smidt HJ, Lagerstedt AS, Penning LC, Voorhout G, Hazewinkel HA, Grinwis GC, Creemers LB, Meij BP, Dhert WJ: The dog as an animal model for intervertebral disc degeneration? Spine (Phila Pa 1976) 2012, 37:351-358.

4. Masuda K, Aota Y, Muehleman C, Imai Y, Okuma M, Thonar EJ, Andersson $\mathrm{GB}, \mathrm{An} \mathrm{HS}$ : A novel rabbit model of mild, reproducible disc degeneration by an annulus needle puncture: correlation between the degree of disc injury and radiological and histological appearances of disc degeneration. Spine (Phila Pa 1976) 2005, 30:5-14.

5. Xia M, Zhu Y: Fibronectin fragment activation of ERK increasing integrin $a_{5}$ and $\beta_{1}$ subunit expression to degenerate nucleus pulposus cells. J Orthop Res 2011, 29:556-561.

6. Haschtmann D, Stoyanov JV, Gédet P, Gédet P, Ferguson SJ: Vertebral endplate trauma induces disc cell apoptosis and promotes organ degeneration in vitro. Eur Spine J 2008, 17:289-299.

7. Grunhagen T, Wilde G, Soukane DM, Shirazi-Adl SA, Urban JP: Nutrient supply and intervertebral disc metabolism. J Bone Joint Surg Am 2006, 88(suppl 2):30-35.

8. Hassan Y, Osman AK, Altyeb A: Noninvasive management of hemangioma and vascular malformation using intralesional bleomycin injection. Ann Plast Surg 2013, 70:70-73.

9. Pienaar C, Graham R, Geldenhuys S, Hudson DA: Intralesional bleomycin for the treatment of hemangiomas. Plast Reconstr Surg 2006, 117:221-226.

10. Wang C, Liu J, Pan W, Wang X, Gao Q, Hou S: Pingyangmycin loaded bovine serum albumin microspheres for chemoembolization therapy-in vitro and in vivo studies. Int J Pharm 2008, 351:219-226.

11. Johannessen W, Auerbach JD, Wheaton AJ, Kurji A, Borthakur A, Reddy R, Elliott DM: Assessment of human disc degeneration and proteoglycan content using T1rhoweighted magnetic resonance imaging. Spine (Phila Pa 1976) 2006, 31:1253-1257.

12. Blumenkrantz G, Zuo J, Li X, Kornak J, Link TM, Majumdar S: In vivo 3.0-tesla magnetic resonance T1rho and T2 relaxation mapping in subjects with intervertebral disc degeneration and clinical symptoms. Magn Reson Med 2010, 63:1193-1200. 
13. Duncan AE, Colman RJ, Kramer PA: Sex differences in spinal osteoarthritis in humans and rhesus monkeys (Macaca mulatta). Spine (Phila Pa 1976) 2012, 37:915-922.

14. Lü DS, Shono Y, Oda I, Abumi K, Kaneda K: Effects of chondroitinase ABC and chymopapain on spinal motion segment biomechanics. An in vivo biomechanical, radiologic, and histologic canine study. Spine (Phila Pa 1976) 1997, 22:1828-1834.

15. Hoogendoorn RJ, Wuisman PI, Smit TH, Everts VE, Helder MN: Experimental intervertebral disc degeneration induced by chondroitinase $A B C$ in the goat. Spine (Phila Pa 1976) 2007, 32:1816-1825.

16. Zhou Z, Jiang B, Zhou Z, Pan X, Sun H, Huang B, Liang T, Ringgaard S, Zou $X$ : Intervertebral disk degeneration: T1 $\rho$ MR imaging of human and animal models. Radiology 2013, 268:492-500.

17. Farndale RW, Buttle DJ, Barrett AJ: Improved quantitation and discrimination of sulphated glycosaminoglycans by use of dimethylmethylene blue. Biochim Biophys Acta 1986, 883:173-177.

18. Wang SZ, Rui YF, Tan Q, Wang C: Enhancing intervertebral disc repair and regeneration through biology: platelet-rich plasma as an alternative strategy. Arthritis Res Ther 2013, 15:220.

19. Benneker LM, Andersson G, latridis JC, Sakai D, Härtl R, Ito K, Grad S: Cell therapy for intervertebral disc repair: advancing cell therapy from bench to clinics. Eur Cell Mater 2014, 27:5-11.

20. Ren S, Liu Y, Ma J, Liu Y, Diao Z, Yang D, Zhang X, Xi Y, Hu Y: Treatment of rabbit intervertebral disc degeneration with co-transfection by adeno-associated virus-mediated SOX9 and osteogenic protein-1 double genes in vivo. Int J Mol Med 2013, 32:1063-1068

21. Xi Y, Kong J, Liu Y, Wang Z, Ren S, Diao Z, Hu Y: Minimally invasive induction of an early lumbar disc degeneration model in rhesus monkeys. Spine (Phila Pa 1976) 2013, 38:E579-E586.

22. Rodriguez AG, Slichter CK, Acosta FL, Rodriguez-Soto AE, Burghardt AJ, Majumdar S, Lotz JC: Human disc nucleus properties and vertebral endplate permeability. Spine (Phila Pa 1976) 2011, 36:512-520.

23. Bibby SR, Fairbank JC, Urban MR, Urban JP: Cell viability in scoliotic discs in relation to disc deformity and nutrient levels. Spine (Phila Pa 1976) 2002, 27:2220-2228.

24. Anderson DG, Tannoury C: Molecular pathogenic factors in symptomatic disc degeneration. Spine J 2005, 5(6 suppl):260S-266S.

25. Hirata H, Yurube T, Kakutani K, Maeno K, Takada T, Yamamoto J, Kurakawa T, Akisue T, Kuroda R, Kurosaka M, Nishida K: A rat tail temporary static compression model reproduces different stages of intervertebral disc degeneration with decreased notochordal cell phenotype. J Orthop Res 2014, 32:455-463.

26. Cs-Szabo G, Ragasa-San Juan D, Turumella V, Masuda K, Thonar EJ, An HS: Changes in mRNA and protein levels of proteoglycans of the anulus fibrosus and nucleus pulposus during intervertebral disc degeneration. Spine (Phila Pa 1976) 2002, 27:2212-2219.

27. Weiler C, Nerlich AG, Zipperer J, Bachmeier BE, Boos N: SSE Award Competition in Basic Science: expression of major matrix metalloproteinases is associated with intervertebral disc degradation and resorption. Eur Spine J 2002, 2002(11):308-320.

28. Millward-Sadler SJ, Costello PW, Freemont AJ, Hoyland JA: Regulation of catabolic gene expression in normal and degenerate human intervertebral disc cells: implications for the pathogenesis of intervertebral disc degeneration. Arthritis Res Ther 2009, 11:R65

29. Bachmeier BE, Nerlich AG, Weiler C, Paesold G, Jochum M, Boos N: Analysis of tissue distribution of TNF-alpha, TNF-alpha-receptors, and the activating TNF-alpha-converting enzyme suggests activation of the TNF-alpha system in the aging intervertebral disc. Ann N Y Acad Sci 2007, 1096:44-54.

30. Hoyland JA, Le Maitre C, Freemont AJ: Investigation of the role of IL-1 and TNF in matrix degradation in the intervertebral disc. Rheumatology (Oxford) 2008, 47:809-814.

31. Burke JG, Watson RW, McCormack D, Dowling FE, Walsh MG, Fitzpatrick JM: Intervertebral discs which cause low back pain secrete high levels of proinflammatory mediators. J Bone Joint Surg Br 2002, 84:196-201.

32. Magalhães J, Ascensão A, Soares JM, Ferreira R, Neuparth MJ, Marques F, Duarte JA: Acute and severe hypobaric hypoxia increases oxidative stress and impairs mitochondrial function in mouse skeletal muscle. J Appl Physiol (1985) 2005, 99:1247-1253.

33. Cervellati F, Cervellati C, Romani A, Cremonini E, Sticozzi C, Belmonte G, Pessina F, Valacchi G: Hypoxia induces cell damage via oxidative stress in retinal epithelial cells. Free Radic Res 2014, 48:303-312.
34. Guehring T, Wilde G, Sumner M, Grünhagen T, Karney GB, Tirlapur UK, Urban JP: Notochordal intervertebral disc cells: sensitivity to nutrient deprivation. Arthritis Rheum 2009, 60:1026-1034

35. Thompson JP, Oegema TR Jr, Bradford DS: Stimulation of mature canine intervertebral disc by growth factors. Spine (Phila Pa 1976) 1991, 16:253-260

36. Lee S, Moon CS, Sul D, Lee J, Bae M, Hong Y, Lee M, Choi S, Derby R, Kim BJ, Kim J, Yoon JS, Wolfer L, Kim J, Wang J, Hwang SW, Lee SH: Comparison of growth factor and cytokine expression in patients with degenerated disc disease and herniated nucleus pulposus. Clin Biochem 2009, 42:1504-1511.

37. Blaney Davidson EN, Remst DF, Vitters EL, van Beuningen HM, Blom AB, Goumans MJ, van den Berg WB, van der Kraan PM: Increase in ALK1/ALK5 ratio as a cause for elevated MMP-13 expression in osteoarthritis in humans and mice. J Immunol 2009, 182:7937-7945.

38. Yamanishi Y, Boyle DL, Clark M, Maki RA, Tortorella MD, Arner EC, Firestein GS: Expression and regulation of aggrecanase in arthritis: the role of TGF-beta. J Immunol 2002, 168:1405-1412.

39. Heathfield SK, Le Maitre CL, Hoyland JA: Caveolin-1 expression and stress-induced premature senescence in human intervertebral disc degeneration. Arthritis Res Ther 2008, 10:R87.

40. Smolders LA, Meij BP, Onis D, Riemers FM, Bergknut N, Wubbolts R, Grinwis GC, Houweling M, Groot Koerkamp MJ, van Leenen D, Holstege FC, Hazewinkel HA, Creemers LB, Penning LC, Tryfonidou MA: Gene expression profiling of early intervertebral disc degeneration reveals a down-regulation of canonical Wnt signaling and caveolin-1 expression: implications for development of regenerative strategies. Arthritis Res Ther 2013, 15:R23.

41. Odajima N, Betsuyaku T, Nasuhara Y, Nishimura M: Loss of caveolin-1 in bronchiolization in lung fibrosis. J Histochem Cytochem 2007, 55:899-909.

42. Battié MC, Videman T: Lumbar disc degeneration: epidemiology and genetics. J Bone Joint Surg Am 2006, 88(suppl 2):3-9.

doi:10.1186/1471-2474-15-340

Cite this article as: Wei et al:: In vivo experimental intervertebral disc degeneration induced by bleomycin in the rhesus monkey. BMC Musculoskeletal Disorders 2014 15:340.

\section{Submit your next manuscript to BioMed Central and take full advantage of:}

- Convenient online submission

- Thorough peer review

- No space constraints or color figure charges

- Immediate publication on acceptance

- Inclusion in PubMed, CAS, Scopus and Google Scholar

- Research which is freely available for redistribution 